\title{
Análise comparativa do pico de consumo de oxigênio entre nadadoras e não nadadoras
}

\author{
Emilson Colantonio ${ }^{1}$ \\ Ronaldo V. Barros \\ Maria Augusta Kiss ${ }^{2}$
}

${ }^{1}$ Centro de Estudos em Psicobiologia e Exercício (CEPE) Associação Fundo de Incentivo a Psicofarmacologia (AFIP)

${ }^{2}$ Laboratório de Determinantes Energéticos de Desempenho Esportivo (LaDESP)

Escola de Educação Física e Esporte

Universidade de São Paulo

Brasil

\section{RESUMO}

Os objetivos do presente estudo foram: a) Comparar a variação dos valores do $\mathrm{VO}_{2 \text { pico }}$ entre diferentes grupos etários de meninas; b) Comparar a variação dos valores do $\mathrm{VO}_{2 \text { pico }}$ (absoluto e relativo), entre nadadoras (Nad) e não nadadoras ( $\mathrm{NNad}$ ) para os mesmos agrupamentos de idade. Foram avaliadas 74 meninas entre 7-17 anos de idade (34Nad e 40NNad), distribuídas em grupos etários de 7-10, 11-14 e 15-17 anos, formando 06 subgrupos, respectivamente: Nad1 $(n=13) ; \operatorname{Nad} 2(n=12)$; Nad3 ( $n=09)$; NNad1 $(n=12)$; NNad2 $(n=14)$; NNad3 $(\mathrm{n}=14)$. Para a obtenção dos valores de $\mathrm{VO}_{2 \text { pico }}$ foi utilizado o sistema de análise de gases $\mathrm{VO} 2000 \AA$ e uma esteira rolante Inbrasport ATL ${ }^{\circledR}$, adotando o protocolo de Bruce adaptado. Os resultados indicaram que houve um aumento do $\mathrm{VO}_{2 \text { pico }}$ absoluto entre todos os grupos etários tanto para $\mathrm{NNad}$ quanto para $\mathrm{Nad}$, o que não foi observado nos valores de $\mathrm{VO}_{2 \text { pico }}$ relativo, sendo que as Nad sempre apresentaram valores superiores às NNad. Tal fato sugere que esse comportamento seja resultado do desenvolvimento natural das avaliadas além do efeito do treinamento de natação, e ainda, que o $\mathrm{VO}_{2 \text { pico }}$ relativo pode não ser um indicador interessante para avaliar a aptidão aeróbia de crianças e jovens.

Palavras-chave: valores metabólicos, crianças e jovens, atletas e não atletas

\section{ABSTRACT \\ Comparative analysis between female swimmers and non-swim- mers in peak oxygen uptake}

The main purpose of the present study was: a) to compare the variation values $V O_{2 \text { peak }}$ variation between different female age-groups; $b$ ) to compare $V_{2 \text { peak }}$ values (absolute and relative), between female swimmers (Sw) and non-swimmers (NSw) for the same age-group. The sample was composed by seventy four female volunteers, ranging in age from 7 to 17 years ( $34 S_{w}$ e 40NSw), and grouped in different ages: 7-10; 11-14; and 15-17 years, making six under groups: three groups composed entirely by female swimmers ( $S w 1, n=13$; Sw2, $n=12$; Sw3, $n=09$ ), and three groups composed by non swimmers (NSw1, $n=12$; NSw2, $n=14$; NSw3, $n=14)$, respectively. The $V O_{2 \text { peak }}$ values were attained by the $\mathrm{VO} 2000{ }^{\circledR}$ gas analysis system and Inbrasport ATL ${ }^{\circledR}$ treadmill, using adapted Bruce protocol. The results pointed out an increment on absolute $V O_{2 p e a k}$ values among the all age-groups considering NSw and Sw groups. The same was not observed for relative $V O_{2 \text { peak }}$ values. The $S w$ always presented higher values in comparison with NSw. These findings suggest that this behavior is a result of natural development of the subjects' more than swimming training. The results also suggest, that relative $V_{2 \text { peak }}$ can not to be considered a very pertinent to evaluate the aerobic fitness of children and adolescents.

Key-words: metabolic values, children and adolescents, athletes and non-athletes 


\section{INTRODUÇÃO}

De acordo com a literatura, é mister o reconhecimento de que o estímulo à prática das atividades físicas por parte de crianças e jovens é uma das necessidades básicas durante o período de crescimento. Entretanto, os programas dessa natureza para esse grupo variam desde atividades recreacionais dirigidas até programas intensivos de esportes competitivos(1).

Alguns autores têm mostrado que o número de competições infanto-juvenis vem aumentando significantemente nas duas últimas décadas $(1,2,3,4,5,6)$. Tal fato tem contribuído para a obtenção de recordes mundiais, especialmente em natação, já aos 14 anos de idade. Sendo assim, com o prestígio atual associado ao sucesso atlético no âmbito nacional e internacional, um grande número de crianças cada vez mais jovens têm se engajado em programas de treinamento esportivo de extrema intensidade.

Os efeitos desses programas na dinâmica do crescimento de crianças e jovens e o quanto as mesmas estão prontas para suportar o rigor desses intensos programas esportivos são questões complexas, já que devem ser considerados múltiplos fatores como o tipo de esporte, a natureza e a freqüência da competição, a idade na qual a atividade foi iniciada, o nível de desenvolvimento da criança e a intensidade e duração do treinamento ${ }^{(7,8,9)}$.

É evidente o aumento do conhecimento das respostas metabólicas e funcionais ao exercício e ao esporte em adultos normais e patológicos ${ }^{(10,11)}$, mas existem algumas lacunas relacionadas ao treinamento físico em crianças e adolescentes $(6,12,13,14)$.

Existem evidências associando atletas de modalidades esportivas, normalmente de endurance, e valores elevados de consumo máximo de oxigênio $\left(\mathrm{VO}_{2 \max }\right)(15,16,17,18,19)$. Dentre as várias modalidades esportivas, é comum encontrarmos também atletas de natação com essa característica metabóli$\mathrm{ca}^{(15,20,21,22)}$, apesar de cerca de $80 \%$ de todos os eventos dos programas das competições são de 200 $\mathrm{m}$ ou menos, isto é, abaixo de dois minutos de duração. Portanto, o treinamento em velocidades máximas se faz necessário para que ocorram as devidas adaptações necessárias na utilização da energia pela via anaeróbia(23).
Visto que nas provas de natação existe importante participação do metabolismo anaeróbio(24), é intrigante pensar porque esses atletas alcançam valores tão elevados de $\mathrm{VO}_{2 \max }$, em relação àqueles atletas de outras modalidades esportivas predominantemente aeróbias.

Investigações realizadas com crianças e adolescentes não atletas em estudos transversais têm mostrado uma tendência de aumento anual de 11,6\% entre oito e 13 anos de idade ${ }^{(25)}$. Outros trabalhos mostraram resultados conflitantes $(26,27,28)$, o que foi corroborado em estudos longitudinais(29).

Portanto, não encontramos respostas conclusivas na literatura sobre a evolução da potência aeróbia durante a infância e adolescência, sobretudo de crianças e jovens atletas envolvidos com a prática sistemática da modalidade esportiva natação durante vários anos.

\section{OBJETIVO}

Os objetivos do presente estudo são: a) comparar a variação do $\mathrm{VO}_{2 \text { pico }}$ entre os diferentes grupos etários de meninas (efeito do crescimento); b) comparar a variação dos valores de $\mathrm{VO}_{2 \text { pico }}$ absoluto e relativo, entre as nadadoras e não nadadoras (efeito do treino sistemático) para os mesmos agrupamentos de idade.

\section{METODOLOGIA}

A amostra foi composta por 74 crianças e jovens voluntárias, do sexo feminino, compreendendo um período etário entre sete e 17 anos de idade, sendo 34 nadadoras (Nad) e 40 não-nadadoras ( $\mathrm{NNad}$ ), distribuídas em três grupos: 7-10, 11-14 e 15-17 anos, com pelo menos três sujeitos de cada idade. Os dados que caracterizam a amostra como valores médios e desvio padrão das medidas antropométricas E (cm), MC (kg), SDC (mm) estão apresentados na Tabela 1.

O grupo de NNad foi formado por alunas de escolas da rede estadual de ensino da Zona Leste da cidade de São Paulo, as quais apenas participavam das aulas de educação física na escola e não estavam engajadas em nenhum tipo de treinamento esportivo sistematizado (não treinadas). Por outro lado, o grupo de Nad foi formado por crianças e jovens que, além de participar das aulas de educação física na escola, também 
Tabela 1. Valores médios e desvio padrão das medidas antropométricas Estatura $[\varepsilon]$ em $\mathrm{cm}$, Massa Corporal (MC) em kg e Somatória de Dobras Cutâneas (SDC) em mm das Nad e NNad.

\begin{tabular}{lcccc}
\hline & $\mathrm{N}$ & $\mathrm{E}(\mathrm{cm})$ & $\mathrm{MC}(\mathrm{kg})$ & $\mathrm{SDC}(\mathrm{mm})$ \\
\hline Nad1 & 13 & $134,91 \pm 7,83$ & $31,76 \pm 8,49$ & $76,11 \pm 48,72$ \\
\hline Nad2 & 12 & $157,88 \pm 7,66$ & $52,03 \pm 6,98$ & $82,54 \pm 23,45$ \\
\hline Nad3 & 9 & $161,41 \pm 6,92$ & $55,58 \pm 4,12$ & $106,42 \pm 28,86$ \\
\hline NNad1 & 12 & $130,82 \pm 8,71$ & $27,97 \pm 6,39$ & $66,41 \pm 37,44$ \\
\hline NNad2 & 14 & $151,51 \pm 8,92$ & $42,04 \pm 11,22$ & $89,98 \pm 35,01$ \\
\hline NNad3 & 14 & $161,54 \pm 3,50$ & $59,91 \pm 10,58$ & $157,66 \pm 43,00$ \\
\hline
\end{tabular}

Tabela 2. Valores médios de carga externa das Nad por agrupamento de idade: tempo de treino [anos], volume de treino na água (horas / $\mathrm{km}$ ), número de sessões semanais e tipo de treino em seco.

\begin{tabular}{lccccc}
\hline $\begin{array}{l}\text { Agrupamento } \\
\text { de idade }\end{array}$ & $\begin{array}{c}\text { Tempo mínimo } \\
\text { de treino }[\text { anos })\end{array}$ & $\begin{array}{c}\text { Volume de treino } \\
\text { na água }(\mathrm{min})\end{array}$ & $\begin{array}{c}\text { Volume de treino } \\
\text { na água }(\mathrm{km})\end{array}$ & $\begin{array}{c}\mathrm{N}^{0} \text { de sessões } \\
\text { semanais }\end{array}$ & $\begin{array}{c}\text { Tipo de treino } \\
\text { em seco }\end{array}$ \\
\hline 7-10 anos & 1 & $100-120$ & $2-2,5$ & 5 & ginástica \\
\hline $11-14$ anos & 2 & $120-150$ & $4-5$ & 6 & ginástica \\
\hline $15-17$ anos & 4 & $100-120(\mathrm{~m})^{*} 240(\mathrm{t})^{* *}$ & $6-8$ & 12 & ginástica musculação \\
\hline
\end{tabular}

*manhã /**tarde

estavam envolvidas com treinamento regular de natação por pelo menos um ano e faziam parte das equipes de competição de suas agremiações (treinadas). As crianças com idades entre sete e 10 anos estavam envolvidas com a natação a pelo menos três anos e participavam de competições de nível regional e estadual. As jovens com idades entre 11 e 14 anos estavam envolvidas com a natação a pelo menos cinco anos e participavam de competições de nível regional, estadual e nacional. As jovens com idades entre 15 e 17 anos estavam envolvidas com a natação a pelo menos oito anos e participavam de competições de nível regional, estadual, nacional e internacional. A tabela 2 mostra as principais características de carga externa, as quais as Nad eram submetidas, não incluindo os dias em que estavam envolvidas com competições nos finais de semana. Após a submissão e aprovação desse projeto ao Comitê de Ética para Pesquisa Científica, protocolo no. 13031623, as seguintes etapas se sucederam: recrutamento das voluntárias, assinatura do termo de consentimento informado para pesquisa científi- ca, preenchimento de formulário de anamnese, realização de medidas antropométricas de estatura (E); massa corporal (MC) e somatória de sete dobras cutâneas (SDC), eletrocardiograma de repouso (ECG) e teste incremental de esforço. A medida de $\mathrm{E}$ foi obtida através da utilização do estadiômetro fixo Sanny ${ }^{\circledR}$ (American Medical do Brasil, Brasil), para a MC uma balança digital Filizola ${ }^{\circledR}$ (Filizola Balanças Industriais, Brasil) e SDC através do adipômetro Sanny ${ }^{\circledR}$ (American Medical do Brasil, Brasil). Foram avaliadas as seguintes dobras cutâneas: triciptal, biciptal, subescapular, supra-ilíaca, abdominal, coxa e panturrilha medial.

Os valores de $\mathrm{VO}_{2 \text { pico }}$ foram obtidos através do sistema de análise de gases modelo VO2000® acoplado ao software Aerograph ${ }^{\circledR}$ e a esteira modelo Inbrasport $A T L{ }^{\circledR}$ (Inbrasport, Brasil). A freqüência cardíaca (FC) e os sinais elétricos do ECG em repouso e durante o exercício foram obtidos através do eletrocardiógrafo modelo Ergo PC 13 Micromed ${ }^{\circledR}$ (Micromed Sistemas, Brasil), utilizando o protocolo adaptado de Bruce et al. (30), seguindo os procedi- 
Tabela 3. Valores médios e desvio padrão de $\mathrm{VO}_{2 \text { pico }}$ absoluto [ $\left(\mathrm{min}^{-1}\right.$ ] e relativo $\left(\mathrm{ml}^{\mathrm{k}} \mathrm{kg}^{-1} \cdot \mathrm{min}^{-1}\right)$.

\begin{tabular}{llcc}
\hline & $\mathrm{N}$ & $\mathrm{VO}_{2 \text { pico }}$ absoluto $\left(\mathrm{L} \cdot \mathrm{min}^{-1}\right)$ & $\mathrm{VO}_{2 \text { pico }}$ relativo $\left(\mathrm{ml}^{\mathrm{k}} \mathrm{kg}^{-1} \cdot \mathrm{min}^{-1}\right.$ ) \\
\hline Nad1 & 13 & $1,18 \pm 0,28$ & $37,77 \pm 6,85$ \\
\hline Nad2 & 12 & $2,21 \pm 0,40$ & $42,61 \pm 6,35$ \\
\hline Nad3 & 9 & $2,42 \pm 0,45$ & $43,42 \pm 6,80$ \\
\hline NNad1 & 12 & $0,94 \pm 0,30$ & $33,40 \pm 6,85$ \\
\hline NNad2 & 14 & $1,38 \pm 0,37$ & $33,24 \pm 4,55$ \\
\hline
\end{tabular}

Tabela 4. Variação em percentual [\%] dos valores médios de $\mathrm{VO}_{2 \text { pico }}$ absoluto e relativo entre os diferentes agrupamentos de idade para Nad e NNad e entre os diferentes grupos (Nad e NNad) para a mesma idade.

\begin{tabular}{|c|c|c|}
\hline & $\mathrm{VO}_{2 \text { pico }}$ absoluto [\%] & $\mathrm{VO}_{2 \text { pico }}$ relativo [\%] \\
\hline Nad3 x Nad1 & 51,23 & 14,95 \\
\hline Nad2 x Nad1 & 46,60 & 11,35 \\
\hline $\mathrm{Nad} 3 \times \mathrm{Nad} 2$ & 8,67 & 1,86 \\
\hline NNad3 $\times$ NNad1 & 48,63 & $-8,93$ \\
\hline NNad2 x NNad1 & 31,88 & $-0,48$ \\
\hline NNad3 $\times$ NNad2 & 24,59 & $-8,41$ \\
\hline Nad1 x NNad1 & 25,53 & 13,08 \\
\hline Nad2 $\times$ NNad2 & 60,14 & 28,18 \\
\hline Nad3 $x$ NNad3 & 32,24 & 41,61 \\
\hline
\end{tabular}

mentos: 1 . aquecimento: velocidade inicial de 2,7 $\mathrm{km} / \mathrm{h}$ e inclinação de $10 \%$ durante três minutos; 2 . sobrecarga: incrementos de $1 \mathrm{~km} / \mathrm{h}$ e $2 \%$ de inclinação a cada minuto até a exaustão. Os critérios de interrupção do teste foram: aumento da pressão arterial sistólica de forma abrupta; aumento desproporcional da pressão arterial diastólica; alterações no ECG; valores de freqüência cardíaca próximas da máxima estimada; exaustão conforme o avaliado. Os testes foram realizados em ambiente exclusivo para esse procedimento com condições ambientais de laboratório estáveis no que se refere à temperatura $\left(21^{\circ} \mathrm{C}\right)$ e pressão barométrica. Os testes foram realizados sempre no período vespertino coincidente ao horário de treinamento das atletas.

Os dados mostraram-se de acordo com os pré-requisitos de normalidade e homocedasticidade de uma análise paramétrica. Foi utilizada análise estatística ANOVA para medidas repetidas, seguidas pelo teste post hoc de Tukey, quando $\mathrm{p}<0,05$. O pacote estatístico utilizado foi o Statistical Package for Social Sciences (SPSS) for Windows, versão 11.0.

\section{RESULTADOS}

Os dados metabólicos de $\mathrm{VO}_{2 \text { pico }}$ absoluto (1.min-1) e relativo $\left(\mathrm{ml}^{\mathrm{kg}} \mathrm{kg}^{-1} \cdot \mathrm{min}^{-1}\right)$ das Nad e NNad estão apresentados na tabela 3 .

A tabela 4 mostra a variação percentual dos valores médios de $\mathrm{VO}_{2 \text { pico }}$ absoluto e relativo entre os diferentes agrupamentos de idade para Nad e NNad e entre os diferentes grupos (Nad e NNad) para a mesma idade.

A Figura 1 mostra os valores médios de $\mathrm{VO}_{2 \text { pico }}$ absoluto (L. $\min ^{-1}$ ) e as relações encontradas entre os 
grupos etários 7-10, 11-14 e 15-17 anos tanto para Nad quanto para NNad; além das relações encontradas dos valores médios de $\mathrm{VO}_{2 \text { pico }}$ absoluto (L.min-1) para os mesmos grupos etários entre Nad e NNad.

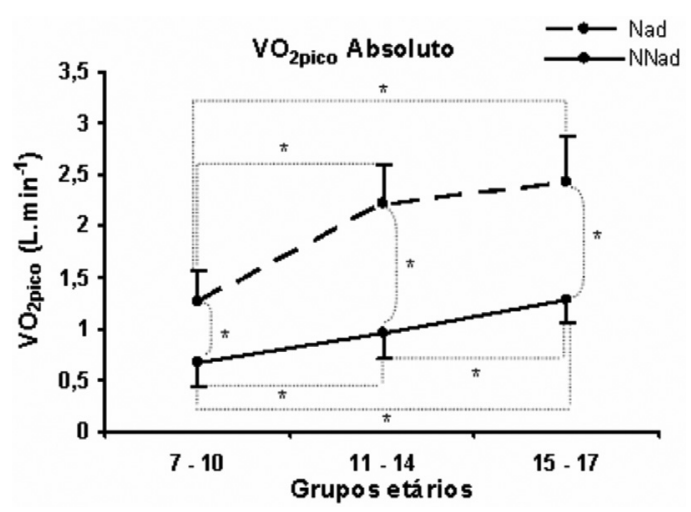

*Indica diferença significante $(p<0,05)$.

Figura 1. Relações entre os valores médios de $\mathrm{VO}_{2 \text { pico }}$ absoluto $\left[\right.$ L. $\mathrm{min}^{-1}$ ] de diferentes grupos etários para Nad e NNad. Relações entre os valores médios de $\mathrm{VO}_{2 \text { ico }}$ absoluto [L.min ${ }^{-1}$ ] para os grupos etários entre Nad e NNad.

A Figura 2 mostra os valores médios de $\mathrm{VO}_{2 \text { pico }}$ relativo $\left(\mathrm{ml} . \mathrm{kg}^{-1} \cdot \mathrm{min}^{-1}\right)$ e as relações encontradas entre os grupos etários 7-10, 11-14 e 15-17 anos tanto para Nad quanto para NNad; além das relações encontradas dos valores médios de $\mathrm{VO}_{2 \text { pico }}$ absoluto (L.min-1) para os mesmos grupos etários entre Nad e NNad.

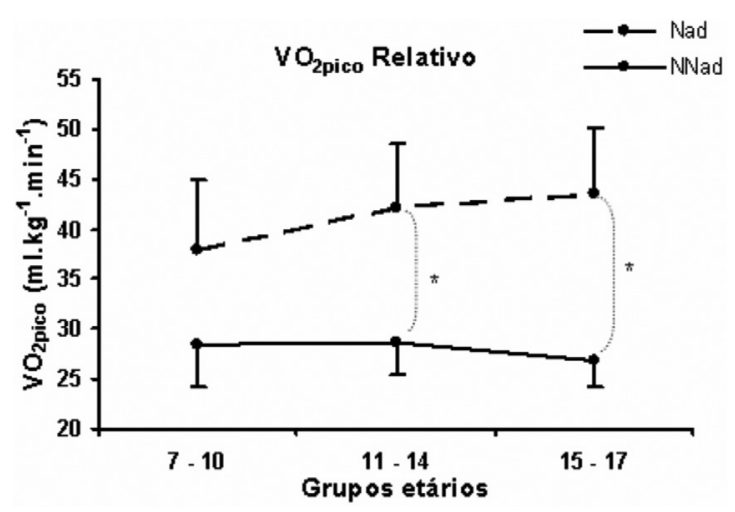

*Indica diferença significante $(p<0,05)$.

Figura 2. Relações entre os valores médios de $\mathrm{VO}_{\text {2pico }}$ relativo $\left(\mathrm{ml} \mathrm{kg}^{-1} \cdot \mathrm{min}^{-1}\right)$ de diferentes grupos etários para Nad e NNad. Relações entre os valores médios de $\mathrm{VO}_{2 \text { ico }}$ relativo ( $\mathrm{ml}^{.} \mathrm{kg}^{-1} . \mathrm{min}^{-1}$ ) para os grupos etários entre Nad e NNad.

\section{DISCUSSÃO}

Diferenças estatísticas significantes foram identificadas nos valores de $\mathrm{VO}_{2 \text { pico }}$ absoluto $\left(1 . \mathrm{min}^{-1}\right)$ entre Nad1 e Nad2, e Nad1 e Nad3. O mesmo comportamento não foi encontrado entre Nad2 e Nad3 $(\mathrm{p}<0.05)$. Com relação aos valores de $\mathrm{VO}_{2 \text { pico }}$ relativo $\left(\mathrm{ml} \cdot \mathrm{kg}^{-1} \cdot \mathrm{min}^{-1}\right)$, não foram observadas diferenças estatísticas significantes entre todos os grupos etários para as Nad.

Diferenças estatísticas significantes foram identificadas nos valores de $\mathrm{VO}_{2 \text { pico }}$ absoluto entre todos os grupos etários de NNad, mas para os valores de $\mathrm{VO}_{2 \text { pico }}$ relativo não foram observadas diferenças estatísticas entre todos os grupos etários de NNad. Os resultados do presente estudo evidenciaram um aumento constante dos valores médios de $\mathrm{VO}_{2 \text { pico }}$ absoluto para todas as faixas etárias independentemente do grupo, isto é, Nad ou NNad. Porém, os maiores valores desta variável para as Nad sempre foram superiores àqueles encontrados para as NNad. Entre as meninas escolares de sete aos 10 anos e dos 11 aos 14 anos de idade a diferença foi de 0,44 \pm 0,07 $1 . \mathrm{m}^{-1}$ e entre 11 a 14 anos e 15 a 17 anos de idade a diferença foi de $0,45 \pm 0,051 . \mathrm{m}^{-1}$. Por outro lado, entre as nadadoras de sete aos 10 anos e dos 11 aos 14 anos de idade a diferença foi de 1,03 \pm $0,121 . \mathrm{m}^{-1}$ e entre 11 a 14 anos e 15 a 17 anos de idade a diferença foi de 0,21 $\pm 0,051 . \mathrm{m}^{-1}$.

Para as meninas, estudos transversais têm mostrado um aumento anual de $11,6 \%$ entre oito e 13 anos com os maiores aumentos entre 11-12 anos e 12-13 anos de idade(25). Outros trabalhos, os resultados indicaram uma manutenção ou queda dessa variável dos 13 aos 15 anos em indivíduos não atletas $(26,27,28)$. Em estudos longitudinais, essas tendências foram confirmadas(29). Outros estudos tanto mostraram que os meninos apresentaram manutenção do $\mathrm{VO}_{2 \max }$ entre 8-16 anos(30), quanto as meninas demonstraram um inequívoco decréscimo dessa variável em função da idade para sujeitos não atletas(31), fato observado também no presente estudo para valores relativos de $\mathrm{VO}_{2 \text { pico }}$ em não atletas. Além do reconhecido aumento do $\mathrm{VO}_{2 \max }$ absoluto com o crescimento, a maior parte da incerteza persiste no entendimento dos fatores pelos quais se estabelece esse aumento. O mais apropriado meio de "normatizar" $\mathrm{o} \mathrm{VO}_{2 \max }$ pelo tamanho corporal pode ser a 
explicação a respeito das diferenças entre gêneros ${ }^{(6)}$. No que se refere aos valores de $\mathrm{VO}_{2 \text { pico }}$ relativo do presente estudo, estes se mostraram inalterados dos sete aos 10 anos de idade e 11 aos 14 anos para as NNad, seguido de um decréscimo não significativo no período dos 11 aos 14 anos e 15 aos 17 anos de idade $\left(-2,58 \pm 0,55 \mathrm{ml} \cdot \mathrm{kg}^{-1} \cdot \mathrm{min}^{-1}\right)$. Para os valores de $\mathrm{VO}_{2 \text { pico }}$ relativo entre as $\mathrm{Nad}$, houve uma tendência de aumento dessa variável para todas as faixas etárias com um maior incremento sendo observado no período dos sete aos 10 anos e 11 aos 14 anos de idade $\left(4,84 \pm 0,72 \mathrm{ml} \cdot \mathrm{kg}^{-1} \cdot \mathrm{min}^{-1}\right)$, em relação ao período de 11 aos 14 anos e 15 aos 17 anos de idade $\left(0,81 \pm 0,35 \mathrm{ml} \cdot \mathrm{kg}^{-1} \cdot \mathrm{min}^{-1}\right)$.

Beunen et al.(32), verificaram que o $\mathrm{VO}_{2 \text { pico }}$ é amplamente explicado pela MC, mas o nível de atividade física e sua interação com o estado maturacional contribuem independentemente com o $\mathrm{VO}_{2 \text { pico, }}$, mesmo depois de a MC ser ajustada. Esses resultados ratificam àqueles encontrados em estudos anteriores que examinaram a influência das diferenças corporais, estado maturacional e a interpretação do $\mathrm{VO}_{2 \max }$ ou $\mathrm{VO}_{2 \text { pico }}(33,34,35,36,37)$.

Os resultados do presente estudo limitam-se aos atletas de natação e escolares investigados considerando semelhanças antropométricas, composição corporal e regime de treinamento. A motivação das voluntárias durante a realização dos testes não foi controlada, apenas incentivada. A variabilidade das condições ambientais foi considerada pequena durante todo o período de testes já que houve controle de temperatura e umidade relativa do ar no ambiente de laboratório. Outro aspecto considerado como limitante foi o fato da não realização de avaliação da maturação biológica das voluntárias devido a grande dificuldade no recrutamento das mesmas e aspectos éticos relacionados à pesquisa científica.

\section{CONSIDERAÇÕES FINAIS}

Os valores médios de $\mathrm{VO}_{2 \text { pico }}$ absoluto entre crianças e jovens de diferentes faixas etárias Nad e NNad não são equivalentes. $\mathrm{O} \mathrm{VO}_{2 \text { pico }}$ relativo parece não ser um bom indicador de aptidão aeróbia para crianças e jovens. Os valores de $\mathrm{VO}_{2 \text { pico }}$ das crianças e jovens de sete a 17 anos de idade Nad e NNad sofrem influência da idade e da MC.

Os achados do presente estudo sugerem que para a amostra estudada o aumento do $\mathrm{VO}_{2 \text { pico }}$ absoluto entre todos os grupos etários entre Nad e NNad é resultado do desenvolvimento biológico natural das voluntárias e provavelmente é evidenciado também pelo efeito do treinamento sistematizado de natação.

\section{CORRESPONDÊNCIA}

\section{Emilson Colantonio}

Rua Marcelo Ribeiro de Mendonça, 27, ap.22, CEP 11030-210

Ponta da Praia, Santos, SP - Brasil

E-mail: nunoec@uol.com.br 


\section{REFERÊNCIAS}

1. Bailey DA, Mirwald RL (1988). The effects of training on growth and development of child. In: Malina RM (ed.), Young Athletes: biological, psychological, and Educational Perspectives. Champaign: Human Kinetics Publishers, 33-47.

2. Bar-Or O (1985). Some notes on physiological and medical considerations for exercises and training of children. In: Binkhordt RA, Kemper HCG, Saris WHM (eds.), Children and Exercise XI. Champaign: Human Kinetics Publishers, 346-53.

3. Colantonio E, Kiss MAPDM (1996). Tópicos de limiar anaeróbio metabólico. Revista Âmbito de Medicina Desportiva, 10: 16-28.

4. Kemper HCG (1995). The Amsterdam growth study: a longitudinal analysis of health, fitness and lifestyle. Champaign: Human Kinetics Publishers.

5. Matveyev LP (1996). Preparação desportiva. Londrina: Centro de Informações Desportivas.

6. Rowland TW (1996). Developmental Exercise Physiology. Champaign: Human Kinetics Publishers.

7. Bailey DA, Malina RM, Mirwald RL (1986). The child, physical activity and growth. In: Falkner F, Tanner JM (eds.), Human Growth: Vol. 2 Postnatal growth, neurology. New York: Plenum, 147-70.

8. Bailey DA, Malina RM, Rasmussen RL (1978). The influence of exercise, physical activity, and athletic performance on the dynamics of human growth. In: Falkner F, Tanner JM (eds.), Human Growth: Vol. 2 Postnatal growth, neurology. New York:: Plenum, 475-505.

9. Malina RM (1983). Human growth, maturation, and regular physical activity. Acta Medica Auxologica, 15: 05-27.

10. Del Nero E, Yazbeck-Jr P, Kedo HH, Kiss MAPDM, Juliano Y, Moffan PJ (1985). Ação do metoprolol duriles na insuficiência coronária crônica. Arquivo Brasileiro de Cardiologia, 45 (03): 211.

11. Negrão CE, Pereira Barreto AC (1998). Efeito do treinamento físico na insuficiência cardíaca: implicações anatômicas, hemodinâmicas e metabólicas. Revista da Sociedade de Cardiologia do Estado de São Paulo, 8(2): 273-84.

12. Bar-Or O (1996). The child and adolescent athlete. Oxford: Blackwell Science.

13. Malina RM, Bouchard C (2002). Atividade Física do Atleta Jovem: do Crescimento à Maturação. São Paulo: Editora Roca.

14. Unnithan VB, Timmons JA, Brogan RT, Paton JY, Rowland TW (1996). Submaximal running economy in run-trained pre-pubertal boys. Journal of Sports Medicine and Physical Fitness, 36(1): 16-23.

15. Dal Monte A, Faina M (1999). Valutazione dell'atleta. Turim: Unione Tipogrrafico-Editrice Torinense.

16. Billat V, Pinoteau J, Petit B (1995). Exercise induced hypoxemia and time to exhaustion at 90,100 and $105 \%$ of the maximal aerobic speed in long distance elite runners. Canadian Journal of Applied Physiology, 20: 102-11.

17. Lavoie NF, Mercer TH (1987). Incremental and constant load determinations of $\mathrm{VO}_{2 \max }$ and maximal constant load. Canadian Journal of Applied Sport Sciences, 12: 229-32.

18. Robergs RA, Roberts SO (1997). Exercise PhysiologyExercise, Performance, and Clinical Applications. Boston: WCB McGraw-Hill.

19. Saltin P, Astrand PO (1967). Maximal oxygen uptake in athletes. Journal of Applied Physiology, 23 (03): 353-8.

20. Eriksson BO, Holmer I, Lundin A (1978). Physiological effects of training in elite swimmers. In: Eriksson BO, Furberg B (eds.), Swimming Medicine IV. Baltimore: University Park Press, 177-87.

21. MacDougall JD, Wenger HA, Green HJ (1991). Physiological testing of the high-performance athlete. Champaign: Human Kinetics Publishers.

22. Wilmore JH, Costill DL (1994). Physiology of Sport and Exercise. Champaign: Human Kinetics Publishers.

23. Troup JP, Trappe TA (1994). Applications of research in swimming. In: Miyashita M, Mutoh Y, Richardson AB (eds.), Medicine and Science in Aquatic Sports, Basel: Karger, 39: 155-65.

24. Gastin PB (2001). Energy system interaction and relative contribution during exercise. Sports Medicine, 31(10): 725-41.

25. Mirwald RL, Bailey DA (1986). Maximal aerobic power. London: Sports Dynamics.

26. Andersen KL, Seliger V, Rutenfranz J, Skrobak-Kaczynski J (1976). Physical performance capacity of children in Norway. Part IV - The rate of growth in maximal aerobic power and the influence of improved physical education of children in a rural community. European Journal of Applied Physiology, 35: 49-58.

27. Armstrong N, Williams J, Balding J, Gentle P, Kirby B (1991). The peak oxygen uptake of British children with reference to age, sex and sexual maturity. European Journal of Applied Physiology and Occupational Physiology, 62 (5): 369-75.

28. Nakagawa A, Ishiko T (1970). Assessment of aerobic capacity with special reference to sex and age of junior and senior high school students in Japan. Japanese Journal of Physiology, 20: 118-29.

29. Armstrong N, Balding J, Gentle P, Kirby B (1990). Patterns of physical activity among 11 to 16 years old British children. British Medical Journal, 301: 203-5.

30. Bruce RA, Kusumi F, Hosmer D (1973). Maximal oxygen intake and nomographic assessment of functional impairment in cardiovascular disease. American Heart Journal, 85: 546-2.

31. Armstrong N, Welsman JR (1994). Assessment and interpretation of aerobic fitness in children and adolescents. Exercise and Sport Sciences Reviews, 22: 435-76.

32. Beunen G, Baxter-jones ADG, Mirwald RL, Thomis M, Lefevre J, Malina RM, Bailey DA (2002). Intraindividual allometric development of aerobic power in 8- to 16-year-old boys. Medicine and Science in Sports and Exercise, 34 (03): 503-10.

33. Armstrong N, Welsman JR (2001). Peak oxygen uptake in relation to growth and maturation in 11- to 17-year-old humans. European Journal of Applied Physiology, 85 (06): 546-51.

34. Eisenmann JC, Pivarnik JM, Malina RM (2001). Scaling peak $\mathrm{VO}_{2}$ to body mass in young male and female distance runners. Journal of Applied Physiology, 90(6): 2172-80.

35. Matecki S, Prioux J, Amsallen F, Mercier J, Prefault C, Ramonatxo M (2001). Maximal oxygen uptake in healthy children: factors of variation and variation standards. Revue des Maladis Respiratories, 18(4): 499-506.

36. Thomis M, Rogers DM, Beunen GP, Woynarowska B, Malina RM (2000). Allometric relationship between body size and peak $\mathrm{VO}_{2}$ related to age at menarche. Annals of Human Biology, 27(6): 623-33.

37. Welsman JR, Armstrong N, Nevill AM, Winter EM, Kirby BJ (1996). Scaling peak $\mathrm{VO}_{2}$ for differences in body size. Medicine and Science in Sports and Exercise, 28(2): 259-65. 